\title{
BMJ Open Potential for the use of mHealth in the management of cardiovascular disease in Kerala: a qualitative study
}

\author{
Rebecca Smith, ${ }^{1}$ Jaideep Menon, ${ }^{2}$ Jaya G Rajeev, ${ }^{3}$ Leo Feinberg, ${ }^{1}$ \\ Raman Krishan Kumar, ${ }^{4}$ Amitava Banerjee ${ }^{5,6}$
}

To cite: Smith R, Menon J, Rajeev JG, et al. Potential for the use of mHealth in the management of cardiovascular disease in Kerala: a qualitative study. BMJ Open 2015;5:e009367 doi:10.1136/bmjopen-2015009367

\section{- Prepublication history and additional material is available. To view please visit the journal (http://dx.doi.org/ 10.1136/bmjopen-2015- 009367).}

Received 14 July 2015

Revised 2 September 2015

Accepted 21 October 2015

\section{CrossMark}

\section{${ }^{1}$ University of Birmingham, Medical School, Birmingham, UK \\ ${ }^{2}$ Department of Preventive Cardiology, Amrita Institute of Medical Sciences \& Research Centre, Kochi, Kerala, India \\ ${ }^{3}$ Government Service, Kochi, Kerala, India \\ ${ }^{4}$ Department of Paediatric Cardiology, Amrita Institute of Medical Sciences \& Research Centre, Kochi, Kerala, India \\ ${ }^{5}$ University of Birmingham Centre for Cardiovascular Sciences, Birmingham, UK ${ }^{6}$ Farr Institute of Health Informatics Research, University College London, London, UK}

Correspondence to Dr Amitava Banerjee; ami.banerjee@ucl.ac.uk

\section{ABSTRACT}

Objectives: To assess the potential for using mHealth in cardiovascular disease (CVD) management in Kerala by exploring: (1) experiences and challenges of current CVD management; (2) current mobile phone use; (3) expectations of and barriers to mobile phone use in CVD management.

Design: Qualitative, semistructured, individual interviews.

Setting: 5 primary health centres in Ernakulam district, Kerala, India.

Participants: 15 participants in total from 3 stakeholder groups: 5 patients with CVD and/or its risk factors, 5 physicians treating CVD and 5 Accredited Social Health Activists (ASHAs). Patients were sampled for maximum variation on the basis of age, sex, CVD diagnoses and risk factors. All participants had access to a mobile phone.

Results: The main themes identified relating to the current challenges of CVD were poor patient disease knowledge, difficulties in implementing primary prevention and poor patient lifestyles. Participants noted phone calls as the main function of current mobile phone use. The expectations of mHealth use are to: improve accessibility to healthcare knowledge; provide reminders of appointments, medication and lifestyle changes; save time, money and travel; and improve ASHA job efficacy. All perceived barriers to mHealth were noted within physician interviews. These included fears of mobile phones negatively affecting physicians' roles, the usability of mobile phones, radiation and the need for physical consultations.

Conclusions: There are three main potential uses of mHealth in this population: (1) as an educational tool, to improve health education and lifestyle behaviours;

(2) to optimise the use of limited resources, by overcoming geographical barriers and financial constraints; (3) to improve use of healthcare, by providing appointment and treatment reminders in order to improve disease prevention and management. Successful mHealth design, which takes barriers into account, may complement current practice and optimise use of limited resources.

\section{BACKGROUND}

mHealth, or mobile health, is the use of mobile devices to support medical and

\section{Strengths and limitations of this study}

- This is the first study linking mHealth and cardiovascular disease in Kerala, which improves our understanding of how mHealth can address population-specific needs.

- These findings are likely to be relevant and generalisable to other rural settings in India.

The study's main limitation is the lack of methodological and investigator triangulation.

public health. ${ }^{1}$ Through the use of existing mobile phone infrastructure, mHealth offers innovative solutions to health problems in high-income economies, where the mHealth industry continues to grow, and also increasingly in low-middle income economies. ${ }^{2} \mathrm{An}$ evaluation of mHealth technologies in developing countries identified a number of key drivers for its implementation, including the empowerment of patients with disease knowledge, overcoming geographical barriers, improved efficiency in data collection and transmission and multilevel cost saving opportunities. ${ }^{3}$ As the second largest subscriber of mobile phones with one of the lowest call tariffs worldwide, ${ }^{4}$ India is an attractive candidate for the mHealth industry. Additionally, India is the fastest growing smartphone market in Asia/Pacific, ${ }^{5}$ facilitating future adoption and development of novel mHealth services.

One of India's most pressing public health concerns is its mounting cardiovascular disease (CVD) burden. By 2020, CVD is projected to become the largest cause of death and disability in India as mortality rates increase to 4.77 million deaths a year. $^{6} 7$ However, the majority of CVD mortality and morbidity is avoidable through a decrease in key modifiable risk factors such as smoking, physical inactivity and dietary intake. ${ }^{8}$ The southern Indian state of Kerala portrays one 
of the poorest CVD risk factor profiles in the nation: $21.9 \%$ are tobacco users, $39.7 \%$ have poor dietary habits, $33.9 \%$ show abdominal obesity, $28.8 \%$ have hypertension, $14.8 \%$ have diabetes mellitus and $54.1 \%$ have hyperlipidaemia. ${ }^{9}$ The consequence of such highly prevalent risk factors is reflected in the results of two separate studies in 2011, both showing CVD as the primary cause of death of adults during the study periods. ${ }^{10}{ }^{11}$ Paradoxically, Kerala's health indicators are favourable compared to the rest of India, including literacy rate (Kerala: $94 \%$, India: $74 \%$ ) and infant mortality rate (Kerala: 12, India: 40/1000 live births), and are similar to those of developed nations. ${ }^{12}$ The causes of Kerala's unexpectedly high CVD prevalence are widely debated, but the urgent need for improved CVD prevention and control is undisputed. ${ }^{713-17}$

Despite the majority of mHealth in low-middle income economies focusing on sexual health and communicable diseases, there is great potential for mHealth to improve CVD management. Existing noncommunicable disease (NCD) mHealth trials have reported improved health outcomes related to NCD management, in particular regarding text message interventions to support exercise, diet and smoking cessation. ${ }^{18-22}$ Other potential benefits of mHealth in a low-resource setting include treatment reminders, disease monitoring and support for patients and health workers. ${ }^{23}{ }^{24}$ In Kerala, a combination of the increasing popularity and economic feasibility of mobile phones, a growing CVD epidemic and a high risk factor prevalence provides a suitable context in which to implement GVD-related mHealth interventions.

Although there is a lack of CVD-related mHealth research in India, quantitative studies have highlighted the likely acceptability and feasibility of mHealth in a rural Indian setting. ${ }^{25}{ }^{26}$ However, owing to the presence of inter-state health disparities, it is important to obtain region-specific data in India; thus, there is still a recognised need for in-depth qualitative research concerning the need and acceptability of mHealth to suit specific populations and develop optimal health interventions. ${ }^{25-28}$ Therefore, this study was designed to investigate the perceptions of three different stakeholder populations in order to assess the potential for using mHealth for CVD management in Kerala. The study objectives are to explore: (1) the experiences and challenges of current CVD management; (2) the current use of mobile phones; (3) the expectations of and barriers to mobile phone use in CVD management.

\section{METHODS}

\section{Context}

The government sector of healthcare in Kerala is structured on primary, secondary and tertiary levels between Ayurveda, Homoeopathy and modern medical systems, the last being the preferred system for specific conditions such as CVD. Primary health centres (PHCs), serving a population of around 10000 , offer health promotion services and activities. ${ }^{29}$ This includes the National Programme for Prevention and Control of Cancer, Diabetes, Cardiovascular Diseases and Stroke, which was implemented in Kerala during 2010-2012 to promote healthy lifestyles, early diagnosis and management of NCDs. ${ }^{30}$ The physicians' roles at PHCs resemble that of a general practitioner and they are responsible for helping patients navigate and utilise higher levels of services or alternative medicines if required.

The National Rural Health Mission employs female community health workers, or Accredited Social Health Activists (ASHAs), to link rural communities with primary healthcare services. Deployed within a village, ASHAs act as the first point of access to healthcare for rural communities. Approximately one ASHA is deployed per thousand population. Their roles include promoting good health practices and providing information on basic determinants of health and existing health services. ${ }^{31}$ The role of ASHAs in communicable disease and maternal health is well established, but there is little evidence concerning their role in NCDs in Kerala. One study assessing ASHA performance motivation highlighted the need for healthcare delivery system improvements, supportive supervision, knowledge enhancement and enabling working modalities, ${ }^{32}$ providing justification for mHealth exploration in this group.

\section{Setting, sample and recruitment}

Following guidance on the qualitative sample size from Guest $e t a l,{ }^{33}$ a minimum sample size of fifteen participants was recruited from five different PHCs across five adjacent village districts in Kerala. The sample consisted of three different stakeholder groups: (1) ASHAs; (2) physicians treating CVD; (3) patients with CVD and/or its risk factors. Maximum variation sampling ${ }^{34}$ of patients, based on age, sex, CVD diagnoses and risk factors, aimed to capture a range of experiences and demographics. Convenience sampling ${ }^{34}$ was suitable for the rest of the sample.

Potential participants meeting the eligibility criteria, which included access to a mobile phone and language fluency in either English or Malayalam, were recruited either by telephone (patients directly) or in person at the PHC (via ASHAs and physicians). Participants nonfluent in English were recruited by a translator fluent in Malayalam and with healthcare experience (doctors or ASHAs); all other participants were recruited by the researcher. One participant from each stakeholder group was recruited from each of the five PHCs, resulting in five ASHAs, five physicians and five patients. All 15 participants initially contacted gave consent to participate.

\section{Data collection}

Individual semistructured interviews took place over a period of 6 weeks. Interview guides covered three topics: (1) the experiences and challenges of current CVD 
management; (2) the current use of mobile phones; (3) expectations of and barriers to mobile phone use in CVD management. Questions were generated using previous qualitative literature examining mHealth applications ${ }^{35-37}$ and were designed to answer the three main research objectives through comprehensive exploration of experiences and views on the topic. Interviews were piloted for relevance, acceptability and understanding for each stakeholder group prior to data collection and adjusted accordingly (see online supplementary appendix 3). Field notes were made immediately after interviews to provide context. All interviews were: (1) with a translator present if necessary; (2) conducted in a private room at the PHC; (3) audio-recorded using a Dictaphone; (4) between 20 and 45 min in length.

\section{Analysis}

Interviews were transcribed verbatim as soon as possible after each interview and uploaded onto NVivo (qualitative data software manager) (RS and JR). Anonymised transcripts were analysed by the researcher (RS) using thematic analysis. ${ }^{38}$ Open coding, which involved thorough familiarisation and annotation of transcript data, occurred over a 2-month period in two phases: (1) immediately after interview transcription; (2) 4 weeks after the last interview transcription. In the first phase, provisional codes were inductively derived from individual data and linked to form themes. Generated codes were recorded in a digital codebook and are displayed in online supplementary appendix 4. Analysis occurred concurrently with data collection to facilitate constant comparison of data-emerging themes were compared to previous individual interview data and between stakeholder group data to identify, develop and confirm overarching themes. Analysis was repeated and checked during the second phase of coding; codes and themes generated on both occasions were compared for agreement and discrepancies were reviewed with respect to the original transcripts in order to ensure rigour and consistency in analysis. In further acknowledgement of the need for transparency and the potential biases introduced by a sole researcher (RS) in data interpretation, reflexivity was practised throughout the entire process. Contradictory evidence was sought to minimise any researcher bias that might interfere with unusual insights. No new codes were added to the codebook after the ninth interview; by definition, ${ }^{39}$ data saturation was achieved.

\section{RESULTS}

All ASHA and physician participants (collectively referred to as 'health workers') were female and aged $<50$ years. As a prerequisite, ASHAs are all literate and of a similar age. ${ }^{31}$ Homogeneity was also observed for physicians working at the PHCs, reasons for which are beyond the scope of this study, but are perhaps explained by Indian cultural norms regarding gender roles. The patient participant characteristics were varied: age range 51-83 years, three males and two females, CVD diagnoses ranging from only one CVD risk factor to more than one acute CVD event. The finalised key themes are categorised under the three interview topic areas and summarised in table 1.

\section{Current experiences and challenges of CVD management}

Although presented separately, the main themes overlap: poor patient disease knowledge, poor implementation of CVD prevention and poor lifestyle. These themes are displayed according to stakeholder group in table 2.

\section{Poor patient disease knowledge}

Twelve of fifteen participants showed low levels of disease awareness and knowledge. Despite existing strategies to increase awareness, the need to further improve patient education was recognised by all stakeholder groups.

"Actually what I think is that there has to be more and
more of health education. Education is the main thing.
Even though we have health education for the people it's

\begin{tabular}{|c|c|c|}
\hline $\begin{array}{l}\text { Current experiences and challenges } \\
\text { of CVD management }\end{array}$ & Current mobile phone use & $\begin{array}{l}\text { Expectations of and barriers to mobile } \\
\text { phone use in CVD management }\end{array}$ \\
\hline 1.1 Poor patient disease knowledge & 2.1 Phone calls & Expectations: \\
\hline $\begin{array}{l}\text { 1.2 Difficulties in implementing primary } \\
\text { prevention }\end{array}$ & 2.2 Ubiquity of mobile phones & $\begin{array}{l}\text { 3.1 Improves accessibility to health information } \\
\text { 3.2 Provides reminders }\end{array}$ \\
\hline 1.3 Poor lifestyle & & 3.3 Saves time, money and travel \\
\hline 1.4 Other: & & 3.4 Improves job efficacy \\
\hline A. Family influence & & Barriers: \\
\hline B. Health worker knowledge support & & 3.5 Usability of mobile phones \\
\hline C. Healthcare expenses & & 3.6 Radiation fears \\
\hline D. Physicians are busy & & 3.7 Potentially negative effect for physicians \\
\hline & & 3.8 Need for physical consultation \\
\hline
\end{tabular}


Table 2 Current experiences and challenges of CVD management

\begin{tabular}{lll}
\hline ASHAs & Physicians & Patients \\
\hline 1.1 Poor patient disease knowledge & 1.1 Poor patient disease knowledge & 1.1 Poor patient disease knowledge \\
1.2 Difficulties in implementing primary & 1.2 Difficulties in implementing primary & 1.3 Poor lifestyle \\
prevention & \multicolumn{1}{c}{ prevention } & 1.4 \\
1.3 Poor lifestyle & 1.3 Poor lifestyle & A. family influence \\
$1.4:$ & $1.4:$ & B. healthcare expenses \\
A. family influence & A. health worker knowledge support & \\
B. health worker knowledge support & B. healthcare expenses & \\
C. healthcare expenses & C. physicians are busy & \\
\hline CVD, cardiovascular disease. &
\end{tabular}

not in effect, they're not actually very much informed." (Physician 3)

"Sometimes I am unsure if I am doing the right thing, like with food, and so I would like advice on that." (Patient 3)

\section{Difficulties implementing primary prevention}

Health workers believed that patients are "not bothered about primary prevention" and are unlikely to implement lifestyle changes and adhere to medication regimes until an acute CVD event occurs. For this reason, they stated that secondary preventative measures are more effective for patients. Participants implied that poor patient disease knowledge is the primary cause for this.

"But most patients, they address the doctor in the last stages ... after they have had an operation, or angiogram, in the hospital, when they feel frightened. This time, when they are scared, they will start controlling the food and exercise. But patients need to be doing this before they have the problem!" (ASHA 2)

\section{Poor lifestyle}

Poor health behaviours were acknowledged to be highly prevalent in this population and a key contributor to CVD development. Moreover, ASHA and physician participants revealed that patients are often slow to practise lifestyle advice and make positive health behaviour changes: they are "busy or working or unavailable" or "tend to forget" lifestyle changes. Participants reported the difficulties of trying to change patient lifestyles, in particular smoking and alcohol use. Health workers thought that patients prefer medication because it is "easier for them" than lifestyle changes.

Diet is a particularly salient point; 8 of 10 health workers discussed the need for better food control in their populations. Participants blamed the increasing availability of fast food, combined with a traditional diet rich in coconut oil and carbohydrates, for causing deteriorating dietary habits. Additionally, urbanisation of formerly rural areas has preceded a rapid shift from daily physical exertion to an increasingly sedentary lifestyle. The detrimental effect of poor diet and lack of exercise is an important influence on CVD development.

“... people did not used to take so much of junk food and such things ... and there's no physical labour, physical exertion. People are mostly doing paperwork. In Kerala, mostly that is the problem.” (Physician 1)

Other

Other notable themes were identified concerning current CVD management:

1. Family influence. Family may be an invaluable support to those patients who are less willing or able to make healthier lifestyle choices.

2. Health worker knowledge support. Health workers would like support in keeping up to date with new guidelines and treatment protocols, especially when working in more remote areas.

3. Healthcare expenses. Patients may avoid having tests and investigations if they cost time, money or travel. Medications not supplied by the government are expensive.

4. Physicians are busy. PHCs are often overcrowded, leaving physicians very little time to spend with each patient.

\section{Current mobile phone use}

Phone calls

Making phone calls was reported as the primary function of mobile phones. Communication between stakeholders is informal and unregulated. In most cases, only those patients requiring more specialised care, such as antenatal and palliative patients, will have their physician's contact number. The main reasons given for mobile phone communication between health workers and patients were for arranging immunisation appointments and home visits and to clarify patient doubts. Communication between health workers can involve calling superiors for advice and patient referrals.

Text messages were used infrequently. One ASHA was concerned that patients literate only in Malayalam would struggle to send and understand text messages on mobile phones, which only accommodate the English alphabet. Text messages can also be an insufficient 
source of information and fail to clarify the recipient's questions. Furthermore, patients reported not being able to understand how to send text messages. The use of internet and email were scarcely mentioned.

\section{Ubiquity of mobile phones}

Participants stated that the vast majority of the population owns, or has access to, a mobile phone, and they have become a necessity in daily life.

"Because most of the patients, everybody, young or old they have the mobile, or at least a basic set." (ASHA 3)

\section{Expectations of and barriers to mobile phone use in CVD management}

The main expectations of and barriers to mobile phone use according to the stakeholder group are summarised in figures 1 and 2.

\section{Improve accessibility to health information}

The communication networks offered by mobile phones could potentially benefit all stakeholders involved by improving accessibility to health information. First, in response to the high risk factor prevalence in this population, mobile phones can be used to disseminate healthy lifestyle advice to support and motivate patients making challenging lifestyle changes.

"[I would like advice] about the food. I think it is good but sometimes I cannot be sure and maybe advice about the food would be helpful." (Patient 2)

Health workers could improve their own clinical knowledge, as well as access decision-making information whenever and wherever they may require it. This removes the geographical and time restraints that are often encountered in busy, remote health centres.
"It's easier because then otherwise you have to ask the patient to come again. I can ask the patient to wait for some time and I contact [a senior] on my phone and get a response immediately, and advise them as they say." (Physician 5)

Data can be shared quickly and efficiently through mobile phones. For example, patients could send in measurements, such as blood glucose readings, without having to travel to the health centre. This enables remote monitoring of disease and treatment adjustment.

\begin{abstract}
"We could send results through mobile phones, like the sugar levels, and the doctor can easily understand what is going on." (ASHA 5)
\end{abstract}

\section{Provide reminders}

Thirteen of fifteen participants reported the potential benefit of receiving reminders about important aspects of disease management, such as appointments, medication and healthy lifestyle changes, in order to improve disease management.

"Actually about fixing appointments, like if they are taking drugs for one month, then if they can get a reminder about getting an appointment ... Actually it can be good if they can get a reminder of drug adherence. If they can get it, that would be good." (Physician 3)

\section{Save time, money and travel}

Although two ASHA participants noted that cumulative use of mobile phones is costly, patients focused on the potential cost-saving opportunities mobile phones could offer.

"For example, if I have to make an appointment, I have to travel to the hospital. So the time, the cost, the travelling, all that is saved with the mobile phone." (Patient 3)
Figure 1 Main expectations of mHealth. ASHSs, Accredited Social Health Activists.

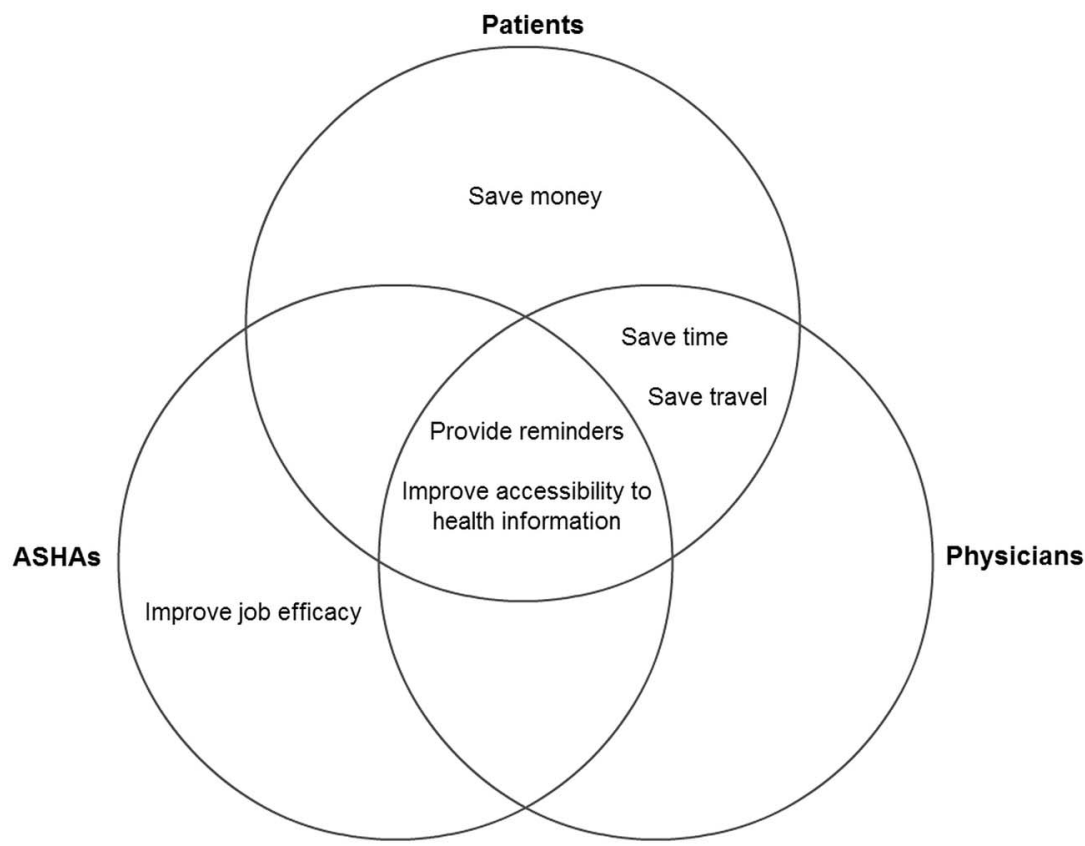


Figure 2 Main barriers to mHealth. ASHSs, Accredited Social Health Activists.

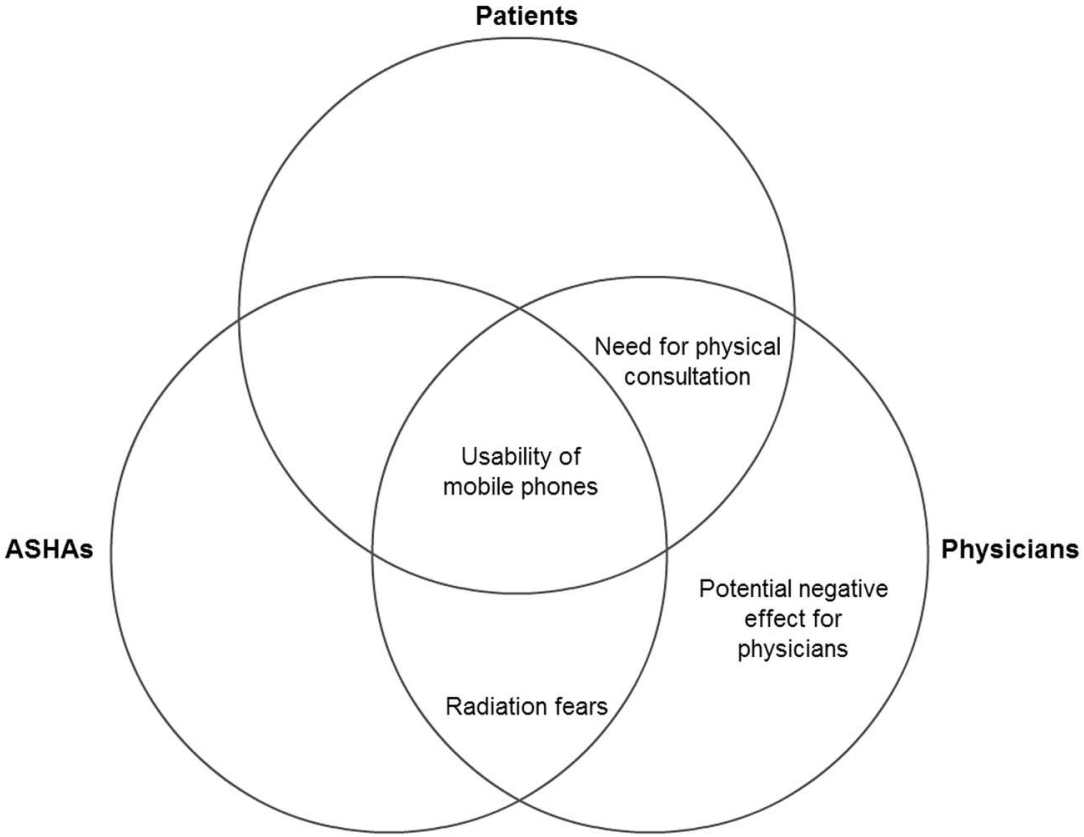

\section{Improve job efficacy}

ASHAs recognised the potential for mobile phone use to improve their work efficacy. The broad reason given was increased communication with patients and colleagues facilitating the planning and conduct of home visits.

"Our work efficiency can be improved. There are more patients who are contacting us, and for us contacting more people on the phone. It would be more useful in many ways." (ASHA 3)

\section{Usability of mobile phones}

Participants acknowledged that you must know how to use the different functions of mobile phones in order to reap any potential benefits. Age was considered to play an important role in this. The younger members of the population are deemed more accepting of mobile phones and more knowledgeable about its various capabilities.

"The patients are not aware of the function of the mobile phones, especially those above fifty or sixty, those who are elderly, so they do not use it. (Physician 4)

\section{Radiation fears}

Four health workers mentioned that the effects of radiation act as a barrier to mobile phone use. They were concerned about mobile phone overuse and the unintentional harm it may cause to the body.

"Mobile by the ear it is near the head, in the pocket it is near the heart, in the pants it is near the man parts! We worry about this bad radiation. It is not good." (ASHA 2)

\section{Potential negative effect for physicians}

Physicians were the main advocates against the use of mobile phones. With busy work schedules, they were concerned that increased use of mobile phones will become a disturbance to their work and personal lives. Unregulated phone use may appear unprofessional and pose a threat to patient care.

"...but for the doctors I think that maybe their job will be affected. Sometimes the call may be coming when they are with another patient." (Physician 5)

\section{Need for physical consultation}

Physicians still need patients to attend physical consultations at the health centre, rather than virtual consultations using a mobile phone. Increased mobile phone use may result in fewer patients attending appointments and hinder the need for physical patient examination.

\footnotetext{
"If you keep asking them about medicines and all on the phone, then I think they will not turn up to the primary care. You can't believe whatever they say. It depends on your examination of the patient." (Physician 2)
}

\section{DISCUSSION}

\section{Principal findings and implications for practice}

To the best of our knowledge, this is the first qualitative study of the potential of mHealth in India, and emphasises three main uses of mHealth: (1) as an educational tool; (2) to optimise the use of limited healthcare and patient resources; (3) to improve use of healthcare services for disease prevention and management. All stakeholders agreed on the potential of mobile phones as an educational tool for healthcare consumers and providers by improving patient disease knowledge and supporting health worker clinical knowledge. Utilisation of existing mobile phone functions, such as phone calls, text messages and internet, or the introduction of novel methods, such as health apps, could increase the 
accessibility and quality of information available (interestingly, apps were not mentioned in any of the interviews, despite their increasing acclaim in the developed world $^{4041}$ ). Consequently, disease prevention would be enhanced by improved compliance with a healthy lifestyle and other primary preventative measures. mHealth also offers opportunities to save time, money and travel, facilitating the optimisation of limited resources. The unique possibility of providing remote care at minimal cost has been echoed in a literature review of mHealth applications along the cancer continuum. ${ }^{42}$ The use of mHealth to provide reminders to patients about appointments, medication and lifestyle has shown improved health outcomes in previous studies, ${ }^{22}{ }^{43}$ with evidence from participants of forgetfulness, nonattendance and non-adherence, this may play a valuable role in CVD prevention and management.

Physicians are the leading opposition to mHealth implementation, with all four themes regarding barriers arising during physician interviews (figure 2). A lack of physician support hindering widespread mHealth use is mirrored in a 2012 report by the Economist Intelligence Unit. ${ }^{44}$ Concerns over radiation are also of note, but current evidence is inconclusive; a review of the available epidemiological evidence states that mobile phone radiation is 'probably' carcinogenic. ${ }^{45}$ Until definitive evidence on the effects of mobile phone radiation emerges, it is likely to remain a barrier to its use. The usability of mobile phones in relation to age must be acknowledged. Participants show concern over the usefulness of mHealth for chronic diseases, which are typically prevalent in elderly, more technologically challenged patients. Although a valid concern for the current CVD population, only a few years separates the technologically literate generation from becoming the generation at risk of CVD. Thus, usability as a barrier is likely to lose its pertinence over future years.

There are several reasons why the success of potential mHealth use may lie in its ability to complement, rather than replace, existing methods of healthcare delivery. Evidence from the developed world has shown that mobile phones can be an effective complement to usual chronic disease management. ${ }^{22}$ Complementary methods would help to facilitate the acceptability of mHealth in a setting where mobile phone technology is a relatively recent innovation-outright replacement of conventional healthcare methods may deter instead of encourage apprehensive users, as is evident from the interviews. For moral and ethical reasons, mHealth should avoid perpetuating socioeconomic health inequalities: those who cannot afford a mobile phone should not be subjected to a lower standard of care than usual; thus, mHealth should not replace usual care. Finally, as reflected in the physician and patient interviews and supported by the results of a similar study for HIV/AIDS in Uganda, ${ }^{46}$ the importance of face-to-face patient-physician contact in disease management must not be forgotten. Without this, certain signs and the underlying context which might be crucial to diagnosis and management may be difficult to ascertain.

What may be most suitable for this population is an intervention similar to several trials worldwide using a bank of text messages to provide lifestyle advice and health awareness to high-risk individuals. ${ }^{47-49}$ This would target the current challenges of CVD management in Kerala in a manner suiting the limited resources and technological capability, as well as complement current methods of care without being intrusive to health workers.

\section{Strengths and limitations}

The results do not aim to be generalisable, but instead transferable to similar settings within Kerala as a valuable first step towards developing future mHealth interventions. However, our findings are likely to be relevant and generalisable to other rural settings in India, where access to health professionals and healthcare infrastructure is limited, despite the rapidly growing private healthcare sector. The timing of this research is also important, considering the growing interest and feasibility of mHealth applications in India across diverse disease areas. ${ }^{50-53}$

Triangulation, through the use of mixed methodology and multiple data analysers, would strengthen this study by improving validity and producing robust and comprehensive findings. Respondent validation of results may establish the credibility of this research; however, participants are yet to comment on this report at the time of production. The researcher also acknowledges that data saturation is a disputable concept and novel themes may have emerged from further interviews. ${ }^{54}$

\section{Areas for future research}

mHealth may have a role to play in improving CVD management in rural India in several ways, including improving access to healthcare professionals where resources are limited and task-shifting to non-physician health workers. Lifestyle change and patient education could be influenced by enabling easier access to information and by increasing patient networks. A survey in PHCs quantifying the extent and depth of physicians' perceived barriers to mHealth would be useful in prioritising where the need for adaptation and development of mHealth design is the greatest. Although text messages are feasible in a population with a high literacy rate, it is evident that calling is preferred; future research may investigate the acceptability and feasibility of text message versus call-based interventions.

\section{CONCLUSION}

mHealth may be a feasible way to target the current challenges of poor lifestyle and patient education in rural India. Complementary mHealth services may reduce high risk factor prevalence in this setting through health promotion and improved measures of 
primary prevention. Possible uses of mHealth include improving attendance at follow-up appointments and improving adherence to medications in primary and secondary prevention of CVD. Development of future uses of mHealth must address the barriers put forward by physicians in order to gain further support for its implementation.

\section{Twitter Follow Amitava Banerjee at @amibanerjee1}

Acknowledgements The authors acknowledge the help of Antje Lindenmeyer, University of Birmingham, in developing the initial protocol for the study.

Contributors The research and analysis was conceived by AB. RS, LF and JGR conducted the interviews under the supervision of RKK and JM. AL provided qualitative methodological support. $R S$ and $A B$ conducted the analyses. RS produced the initial draft of the manuscript and all authors contributed to the final manuscript.

Funding This study was funded by the Arthur Thompson Trust intercalating travel award at the University of Birmingham and the Amrit Dhoot Award at the South Asian Health Foundation.

Competing interests None declared

Ethics approval This study received ethical approval from the BMedSc Population Sciences and Humanities Internal Ethics Review Committee at the University of Birmingham and the Amrita Institute of Medical Sciences Ethics Committee, India. The study protocol and patient consent form are included in Appendices 1 and 2, respectively.

Provenance and peer review Not commissioned; externally peer reviewed.

Data sharing statement No additional data are available.

Open Access This is an Open Access article distributed in accordance with the Creative Commons Attribution Non Commercial (CC BY-NC 4.0) license, which permits others to distribute, remix, adapt, build upon this work noncommercially, and license their derivative works on different terms, provided the original work is properly cited and the use is non-commercial. See: http:// creativecommons.org/licenses/by-nc/4.0/

\section{REFERENCES}

1. World Health Organisation. mHealth: New horizons for health through mobile technologies: Based on the findings of the second global survey on eHealth. Vol III. Global observatory for eHealth series, 2011.

2. Chib A, van Velthoven $\mathrm{MH}$, Car J. mHealth adoption in low-resource environments: a review of the use of mobile healthcare in developing countries. J Health Commun 2015;20:4-34

3. Marshall C, Lewis D, Whittaker M. mHealth technologies in developing countries: a feasibility assessment and a proposed framework. Health Information Systems Knowledge Hub Working Paper Series, No 25. 2013.

4. Telecom Regulatory Authority of India. TRAI Annual Report 20122013. 2013

5. International Data Corporation. IDC announces India as the fastest growing smartphone market in Asia/Pacific in Q3. 2014. http://www. idc.com/getdoc.jsp?containerld=prIN25276014 (accessed 02/05/15)

6. Ezzati M, Hoorn SV, Lopez AD, et al. Comparative quantification of mortality and burden of disease attributable to selected risk factors. In: Lopez AD, Mathers CD, Ezzati M, et al., eds. Global burden of disease and risk factors. Washington DC, 2006:241-68.

7. Coelho KR. Significance of the development of a cardiovascular disease surveillance and reporting system in India. Indian J Palliat Care 2013;19:131-8.

8. Deaton C, Froelicher ES, Wu LH, et al. The global burden of cardiovascular disease. Eur J Cardiovasc Nurs 2011;10(Suppl 2): S5-13.

9. Thankappan KR, Shah B, Mathur P, et al. Risk factor profile for chronic non-communicable diseases: results of a community-based study in Kerala, India. Indian J Med Res 2010;131:53-63.

10. Soman CR, Kutty VR, Safraj S, et al. All-cause mortality and cardiovascular mortality in Kerala state of India: results from a 5-year follow-up of 161,942 rural community dwelling adults. Asia Pac J Public Health 2011;23:896-903.

11. Sauvaget C, Ramadas K, Fayette JM, et al. Socio-economic factors \& longevity in a cohort of Kerala State, India. Indian J Med Res 2011;133:479-86.

12. National Rural Health Mission. State wise information: Kerala Ministry of Health and Family Welfare, Government of India. 2013. http://nrhm.gov.in/nrhm-in-state/state-wise-information/kerala.html (accessed 2 Feb 2015).

13. Thresia CU. Rising private sector and falling 'good health at low cost': health challenges in China, Sri Lanka, and Indian state of Kerala. Int J Health Serv 2013;43:31-48.

14. Yadav K, Nikhil S, Pandav CS. Urbanization and health challenges: need to fast track launch of the national urban health mission. Indian J Community Med 2011;36:3-7.

15. Shah B, Mathur P. Surveillance of cardiovascular disease risk factors in India: the need \& scope. Indian J Med Res 2010;132:634-42.

16. Srinath Reddy K, Shah B, Varghese C, et al. Responding to the threat of chronic diseases in India. Lancet 2005;366:1744-9.

17. Wood A, Pell J, Patel A, et al. Prevention of cardiovascular disease in a rural region of India and strategies to address the unmet need. Heart 2011:97:1373-8.

18. Jacobs MA, Cobb CO, Abroms L, et al. Facebook apps for smoking cessation: a review of content and adherence to evidence-based guidelines. J Med Internet Res 2014;16:e205.

19. Cole-Lewis $\mathrm{H}$, Kershaw $\mathrm{T}$. Text messaging as a tool for behavior change in disease prevention and management. Epidemiol Rev 2010;32:56-69.

20. Liu WT, Wang $\mathrm{CH}$, Lin HC, et al. Efficacy of a cell phone-based exercise programme for COPD. Eur Respir J 2008;32:651-9.

21. Anglada-Martinez H, Riu-Viladoms G, Martin-Conde M, et al. Does mHealth increase adherence to medication? Results of a systematic review. Int J Clin Pract 2015;69:9-32.

22. Beratarrechea A, Lee AG, Willner JM, et al. The impact of mobile health interventions on chronic disease outcomes in developing countries: a systematic review. Telemed J E Health 2014;20:75-82.

23. Källander K, Tibenderana JK, Akpogheneta OJ, et al. Mobile Health (mHealth) approaches and lessons for increased performance and retention of community health workers in low- and middle-income countries: a review. J Med Internet Res 2013;15:e17.

24. Arie S. Can mobile phones transform healthcare in low and middle income countries? BMJ 2015;350:h1975.

25. Priyaa S, Murthy S, Sharan S, et al. A pilot study to assess perceptions of using SMS as a medium for health information in a rural setting. Technol Health Care 2014;22:1-11.

26. DeSouza SI, Rashmi MR, Vasanthi AP, et al. Mobile phones: the next step towards healthcare delivery in rural India? PLOS ONE 2014;9:e104895.

27. Purohit BC. Inter-state disparities in health care and financial burden on the poor in India. J Health Soc Policy 2004;18:37-60.

28. Davey S, Davey A, Singh JV. Mobile-health approach: a critical look on its capacity to augment health system of developing countries. Indian J Community Med 2014;39:178-82.

29. Government of Kerala. Health Policy Kerala 2013. Health and Family Welfare Department. http://dhs.kerala.gov.in/docs/draftpolicy. pdf (accessed 2 Feb 2015)

30. National Rural Health Mission. National Programme for Prevention and Control of Cancer, Diabetes, Cardiovascular Diseases and Stroke (NPCDCS). Ministry of Health and Family Welfare, Government of India. 2010. http://www.nrhmhp.gov.in/sites/default/ files/files/NCD_Guidelines.pdf (accessed 2 Feb 2015).

31. National Rural Health Mission. About Accredited Social Health Activist (ASHA). Ministry of Health and Family Welfare, Government of India. 2013. http://nrhm.gov.in/communitisation/asha/about-asha. html (accessed 2 Feb 2015).

32. Gopalan SS, Mohanty S, Das A. Assessing community health workers' performance motivation: a mixed-methods approach on India's Accredited Social Health Activists (ASHA) programme. BMJ Open 2012;2:pii: e001557.

33. Guest G, Bunce A, Johnson L. How many interviews are enough?: an experiment with data saturation and variability. Field Methods 2006;18:59-82.

34. Luborsky MR, Rubinstein RL. Sampling in Qualitative Research: Rationale, Issues, and Methods. Res aging 1995;17:89-113.

35. Williams V, Price J, Hardinge $M$, et al. Using a mobile health application to support self-management in COPD: a qualitative study. Br J Gen Pract 2014;64:e392-400.

36. Burner ER, Menchine MD, Kubicek K, et al. Perceptions of successful cues to action and opportunities to augment behavioral triggers in diabetes self-management: qualitative analysis of a 
mobile intervention for low-income Latinos with diabetes. J Med Internet Res 2014;16:e25.

37. Surka S, Edirippulige S, Steyn K, et al. Evaluating the use of mobile phone technology to enhance cardiovascular disease screening by community health workers. Int J Med Inform 2014;83:648-54.

38. Braun V, Clarke V. Using thematic analysis in psychology. Qual Res Psychol 2006;3:77-101.

39. Seale C. Grounding theory. In: Seale C, ed. The quality of qualitative research. London: SAGE Publications Ltd, 1999:87-105.

40. Payne $\mathrm{HE}$, Lister $\mathrm{C}$, West $\mathrm{JH}$, et al. Behavioral functionality of mobile apps in health interventions: a systematic review of the literature. JMIR mHealth UHealth 2015;3:e20.

41. Becker S, Miron-Shatz T, Schumacher N, et al. mHealth 2.0: Experiences, Possibilities, and Perspectives. JMIR MHealth UHealth 2014;2:e24-00.

42. Davis SW, Oakley-Girvan I. mHealth education applications along the cancer continuum. J Cancer Educ 2015;30:388-94.

43. Kannisto KA, Koivunen MH, Välimäki MA. Use of mobile phone text message reminders in health care services: a narrative literature review. J Med Internet Res 2014;16:e222.

44. The Economist Intelligence Unit and PricewaterhouseCoopers. Emerging mHealth: Paths for growth. 2014. http://www.pwc.com/en GX/gx/healthcare/mhealth/assets/pwc-emerging-mhealth-full.pdf (accessed 2 Feb 2015)

45. Morgan LL, Miller AB, Sasco A, et al. Mobile phone radiation causes brain tumors and should be classified as a probable human carcinogen (2A) (Review). Int J Oncol 2015;46:1865-71.

46. Chang LW, Njie-Carr V, Kalenge S, et al. Perceptions and acceptability of $\mathrm{mHealth}$ interventions for improving patient care at a community-based HIV/AIDS clinic in Uganda: a mixed methods study. AIDS Care 2013;25:874-80.

47. Redfern J, Thiagalingam A, Jan S, et al. Development of a set of mobile phone text messages designed for prevention of recurrent cardiovascular events. Eur J Prev Cardiol 2014;21:492-9.

48. Albright $\mathrm{K}$, Krantz MJ, Backlund Jarquin $\mathrm{P}$, et al. Health promotion text messaging preferences and acceptability among the medically underserved. Health Promot Pract 2015;16:523-32.

49. Lin $\mathrm{PH}$, Wang $\mathrm{Y}$, Levine $\mathrm{E}$, et al. A text messaging-assisted randomized lifestyle weight loss clinical trial among overweight adults in Beijing. Obesity (Silver Spring) 2014;22:E29-37.

50. Modi D, Gopalan R, Shah S, et al. Development and formative evaluation of an innovative mHealth intervention for improving coverage of community-based maternal, newborn and child health services in rural areas of India. Glob Health Action 2015; 8:26769.

51. Patterson V. Telemedicine for epilepsy support in resource-poor settings. Front Public Health 2014;2:120.

52. Jain N, Singh H, Koolwal GD, et al. Opportunities and barriers in service delivery through mobile phones (mHealth) for Severe Mental Illnesses in Rajasthan, India: a multi-site study. Asian J Psychiatr 2015;14:31-5

53. Raghu A, Praveen D, Peiris D, et al. Engineering a mobile health tool for resource-poor settings to assess and manage cardiovascular disease risk: SMARThealth study. BMC Med Inform Decis Mak 2015;15:36.

54. O’Reilly M, Parker N. 'Unsatisfactory Saturation': a critical exploration of the notion of saturated sample sizes in qualitative research. Qual Res 2013;13:190-7. 